\title{
Effects of Endurance Exercise Training on Markers of Cholesterol Absorption and Synthesis
}

\author{
K. R. WILUND ${ }^{1}$, L. A. FEENEY ${ }^{1}$, E. J. TOMAYKO ${ }^{1}$, E. P. WEISS ${ }^{2}$, J. M. HAGBERG ${ }^{3}$ \\ ${ }^{1}$ Department of Kinesiology and Community Health, University of Illinois, Urbana, IL, \\ ${ }^{2}$ Department of Nutrition and Dietetics, Saint Louis University, St Louis, MO, ${ }^{3}$ Department of \\ Kinesiology, University of Maryland, College Park, MD, USA
}

Received January 25, 2008

Accepted July 1, 2008

On-line July 25, 2008

\section{Summary}

Abnormal cholesterol metabolism, including low intestinal cholesterol absorption and elevated synthesis, is prevalent in diabetes, obesity, hyperlipidemia, and the metabolic syndrome. Diet-induced weight loss improves cholesterol absorption in these populations, but it is not known if endurance exercise training also improves cholesterol homeostasis. To examine this, we measured circulating levels of campesterol, sitosterol, and lathosterol in 65 sedentary subjects (average age 59 years; with at least one metabolic syndrome risk factor) before and after 6 months of endurance exercise training. Campesterol and sitosterol are plant sterols that correlate with intestinal cholesterol absorption, while lathosterol is a marker of whole body cholesterol synthesis. Following the intervention, plant sterol levels were increased by $10 \%(p<0.05)$, but there was no change in plasma lathosterol. In addition, total and LDLcholesterol were reduced by $0.16 \mathrm{mmol}$ and $0.10 \mathrm{mmol}$, respectively $(p<0.05)$, while HDL-C levels increased by $0.09 \mathrm{mmol}(p<0.05)$. Furthermore, the change in plant sterols was positively correlated with the change in $\mathrm{VO}_{2} \max (r=0.310$, $p=0.004$ ), independent of other metabolic syndrome risk factors. These data indicate that exercise training reduces plasma cholesterol despite increasing cholesterol absorption in subjects with metabolic syndrome risk factors.

\section{Key words}

Exercise • Plant sterols • Cholesterol metabolism

\section{Corresponding author}

K. R. Wilund, Department of Kinesiology and Community Health, University of Illinois at Urbana-Champaign, 906 S. Goodwin Avenue, Urbana, IL 61801, USA. E-mail: kwilund@uiuc.edu

\section{Introduction}

Human plasma contains small amounts of noncholesterol sterols that provide information related to cholesterol homeostasis. For example, lathosterol, a precursor in the cholesterol synthetic pathway, is a marker for whole body cholesterol synthesis, while plant sterols, including campesterol and sitosterol, correlate with rates of cholesterol absorption (Miettinen et al. 1990). Furthermore, the ratio between campesterol and lathosterol is often used to indicate the ratio between cholesterol absorption and synthesis (Miettinen et al. 1990).

Individuals with type II diabetes, hyperlipidemia, and obesity have altered cholesterol homeostasis, indicated by low cholesterol absorption and/or elevated cholesterol synthesis (Chan et al. 2003, Gylling and Miettinen 1997, Miettinen et al. 2004, Simonen et al. 2000, Sutherland et al. 1992). Dietinduced weight loss improves many risk factors for the metabolic syndrome and was recently shown to increase cholesterol absorption in obese diabetics (Simonen et al. 2000). Furthermore, in this study the increase in cholesterol absorption was correlated with improvements in insulin resistance, leading to the suggestion that low cholesterol absorption should be considered a component of the metabolic syndrome (Simonen et al. 2000).

Endurance exercise training also improves traditional metabolic syndrome risk factors, but little is known about the effects of exercise training on cholesterol absorption or synthesis. Two recent studies by Varady et al. $(2004,2007)$ showed that 8 weeks of 
endurance exercise training had little mixed effects on markers of cholesterol absorption and synthesis. However, the relatively short exercise interventions used in these studies may have been insufficient to provide the metabolic adaptations necessary to promote significant changes in cholesterol homeostasis. The purpose of this study was to examine the effect of long-term endurance exercise training on cholesterol absorption and synthesis by measuring circulating plant sterol and lathosterol levels before and after a 6-month endurance exercise training intervention in individuals at risk for developing metabolic syndrome.

\section{Methods}

\section{Subjects}

Sixty-five subjects ( 31 men, 34 women) between the ages of 50-70 years were included in this study. Eligibility requirements included the following: sedentary (defined as participating in $<40$ minutes/week of aerobic activity for the 6 months prior to the study); nonsmoking, free of CVD, non-diabetic, body mass index less than 37, not on lipid or glucose-lowering medications; no history of ulcers or other bleeding disorders, and no other medical conditions that would preclude subjects from participating in a vigorous exercise training program. In addition, all subjects had at least one plasma lipoprotein-lipid abnormality (TG > $200 \mathrm{mg} / \mathrm{dl}(2.2 \mathrm{mmol})$, LDL-C $>120 \mathrm{mg} / \mathrm{dl}$ (3.12 mmol), or HDL-C $<40 \mathrm{mg} / \mathrm{dl}(1.04 \mathrm{mmol})$ for men, or $<45$ $\mathrm{mg} / \mathrm{dl}$ (1.17 mmol) for women, pre-hypertension, or Stage 1 hypertension. Furthermore, all women were postmenopausal and were required to maintain their current hormone replacement regimen (on or not on) for the duration of the study. The experimental protocol was approved by the University of Maryland - College Park Institutional Review Board, and all subjects provided their written informed consent prior to starting the study.

\section{Dietary control}

All subjects meeting the preliminary requirements for the study began a 6-week dietary instruction class to stabilize them on an American Heart Association (AHA) step 1 diet prior to baseline testing. Compliance was monitored by the completion of 7-day food records before and after the exercise intervention period. A computerized nutrient analysis using Nutritionist IV software (N-Squared computing, San Bruno, CA), was performed on food records from a subset of the subjects $(\mathrm{n}=30)$ to determine whether dietary patterns that may affect body composition or lipid metabolism (i.e., cholesterol intake, total caloric intake, and the percentage of calories from total fat, saturated fat, protein, and carbohydrate) differed at baseline or final testing. In addition, subjects were instructed to maintain their caloric intake throughout the intervention period to prevent excessive weight loss. Subject's who lost more than $0.5-1.0 \mathrm{~kg}$ per month (the expected weight loss based on caloric expenditure from the exercise) were instructed to increase their caloric intake to ensure that changes in physiological variables were due to changes in physical activity, and not diet-induced weight loss.

\section{$\mathrm{VO}_{2}$ max testing}

After subjects were stabilized on the AHA step I diet, all subjects had their $\mathrm{VO}_{2}$ max determined during graded treadmill walking or jogging using a modified protocol with the grade adjusted $2 \%$ to $3 \%$ every 2 min during the test so that the total exercise time before the subject reached subjective exhaustion would be 8-12 min (Seals et al. 1984). Blood pressure, heart rate, and electrocardiogram were monitored before, during, and after all tests.

\section{Body composition}

Total body fat, lean body mass, and regional body fat were assessed by dual energy X-ray absorptiometry (DEXA) (model DPX-L; Lunar Corp., Madison, WI), as described elsewhere (Mazess et al. 1990). Intra-abdominal (IA) fat (visceral and subcutaneous [SC] adipose tissue areas) was assessed by computed tomography (CT) scan midway between L4 and L5 using a GE HiLight CT scanner (Phillips Medical Systems Philadelphia, PA) (Nicklas et al. 1996)

\section{Plasma lipids}

Venous blood samples were drawn after a 12-h fast for analysis of major plasma lipid concentrations. Baseline samples were drawn at the end of the dietary stabilization program. Plasma was collected by centrifugation and frozen at $-80{ }^{\circ} \mathrm{C}$ until analyzed.

Plasma sterols were measured by gas-liquid chromatography (GLC), using an automated 6890N chromatograph from Agilent Technologies carrying an HP 5MS column, as described (Wilund et al. 2004). Cholesterol and plasma non-cholesterol sterol concentrations were calculated by using calibration curves prepared from known standards. By convention 
(Miettinen et al. 1990), circulating levels of each of these sterols are normalized to plasma cholesterol levels for data presentation.

\section{Fasting glucose and insulin}

Fasting plasma glucose and insulin levels were analyzed using the glucose oxidase method (model 2300 Stat Plus; YSI Inc., Yellow Springs, $\mathrm{OH}$ ) and a radioimmunoassay (kit HI-14K; Linco Research Inc., St. Charles, MO), respectively. The insulin assay was only run on plasma samples from 30 of the 65 subjects analyzed in this study due to limited sample availability.

\section{Exercise training protocol}

Following baseline testing, all subjects began a 6-month exercise training program that consisted of exercising three times per week for 6 months on stairstepping machines, treadmills, or stationary bicycles in the presence of study personnel. Details of the training protocol are described elsewhere (Wilund et al. 2002).

\section{Final testing}

After training, all subjects completed the same plasma lipid, body composition, and $\mathrm{VO}_{2} \max$ assessments as prior to training. The blood samples were drawn 24-36 h after each subjects' prior exercise training session.

\section{Statistics}

All data are presented as the mean \pm S.E.M., and $\mathrm{p}<0.05$ was accepted as statistically significant. Normality of distribution was assessed using Kolmogorov-Smirnov and Shapiro-Wilks' tests. Changes in our primary outcome variables were similar between genders, so the data from all subjects were pooled for analysis. Paired sample t-tests were conducted to examine training related effects on all variables. Pearson correlation analysis was used to examine bivariate relationship between outcomes of interest. Factors related to the change in non-cholesterol sterols (lathosterol or plant sterols) were determined by multiple linear regression in models containing the change in $\mathrm{VO}_{2} \mathrm{max}$ $(\mathrm{ml} / \mathrm{kg} / \mathrm{min})$ and metabolic syndrome risk factors, including blood pressure, and plasma HDL-C, TG, and glucose as independent variables $\left(p_{\text {in }}=0.05, p_{\text {out }}=1.0\right.$ ). All data were analyzed using SPSS v.15.0 (SPSS, Inc).

\section{Results}

Values for our major outcome variables at
Table 1. Subject characteristics before and after 6 months of endurance exercise training.

\begin{tabular}{lll}
\hline Measure & Baseline & Final \\
\hline VO ${ }_{2}$ max (ml/kg/min) & $25.0 \pm 0.6$ & $28.7 \pm 0.69^{*}$ \\
Body weight $(\mathrm{kg})$ & $81.6 \pm 2.1$ & $80.4 \pm 2.0^{*}$ \\
Body fat (\%) & $36.3 \pm 1.1$ & $34.9 \pm 1.1^{*}$ \\
IA fat $\left(\mathrm{cm}^{2}\right)$ & $137 \pm 7.4$ & $128 \pm 6.6^{*}$ \\
SC fat $\left(\mathrm{cm}^{2}\right)$ & $308 \pm 14.6$ & $298 \pm 13.3$ \\
LBM $(\mathrm{kg})$ & $48.5 \pm 1.5$ & $49.1 \pm 1.5^{*}$ \\
Systolic BP (mm Hg) & $130.1 \pm 2.2$ & $133.0 \pm 2.0$ \\
Diastolic BP (mm Hg) & $86.1 \pm 1.3$ & $83.8 \pm 1.4$ \\
Fasting glucose (mmol/l) & $5.08 \pm 0.09$ & $5.28 \pm 0.09^{*}$ \\
Fasting insulin (pmol/l) & $76.4 \pm 4.7$ & $66.7 \pm 3.6^{*}$ \\
Triglycerides (mmol/l) & $1.58 \pm 0.09$ & $1.41 \pm 0.08^{*}$ \\
Total cholesterol (mmol/l) & $5.36 \pm 0.10$ & $5.20 \pm 0.10^{*}$ \\
HDL-C (mmol/l) & $1.19 \pm 0.04$ & $1.28 \pm 0.04^{*}$ \\
LDL-C mmol/l) & $3.35 \pm 0.08$ & $3.25 \pm 0.08^{*}$ \\
Lathosterol/cholesterol & $2.70 \pm 0.17$ & $2.63 \pm 0.15$ \\
x 10 & & \\
Campesterol/cholesterol & $2.42 \pm 0.10$ & $2.74 \pm 0.13^{*}$ \\
x 10 & & \\
Sitosterol/cholesterol x 10 & $1.72 \pm 0.13$ & $1.85 \pm 0.14$ \\
Total plant & $4.14 \pm 0.20$ & $4.58 \pm 0.24^{*}$ \\
sterols/cholesterol x 10 ${ }^{3}$ & & \\
Campesterol/lathosterol & $1.06 \pm 0.08$ & $1.20 \pm 0.08^{*}$ \\
\hline & & \\
\hline & & \\
\hline
\end{tabular}

Data presented are means \pm S.E.M. ${ }^{*} \mathrm{p}<0.05$ compared to baseline.

baseline and final testing are shown in Table $1 . \mathrm{VO}_{2} \max$ increased by $\sim 15 \%$ following the exercise training intervention $(\mathrm{p}<0.001)$, and there was a modest $(1.2 \mathrm{~kg})$ reduction in total body weight $(\mathrm{p}<0.001)$. There was a small $(1.3 \%)$ increase in lean body mass (LBM) $(\mathrm{p}=0.005)$, and $\%$ body fat and intra-abdominal fat mass were reduced by $4 \%(p<0.001)$ and $7 \%(p=0.002)$, respectively. However, there was no significant change in subcutaneous fat mass $(\mathrm{p}=0.10)$. Plasma lipids improved in response to the exercise intervention, as TG, TC and LDL-C were each significantly reduced, while HDL-C increased after the intervention ( $p<0.05$ for each). In addition, fasting plasma insulin levels were reduced by $14 \%$, though plasma glucose levels paradoxically increased by $\sim 10 \%(\mathrm{p}<0.05$ for each $)$.

There was no significant difference in plasma sitosterol or lathosterol levels between baseline and final testing $(p=0.22$ and $p=0.67$, respectively). However, 
Table 2. Correlation coefficients $\left(r\right.$ ) between plasma sterols, $\mathrm{VO}_{2} \max$, and metabolic syndrome risk factors at baseline and final testing.

\begin{tabular}{|c|c|c|c|c|}
\hline & Lathosterol & Campesterol & Sitosterol & Total plant sterols \\
\hline \multicolumn{5}{|c|}{$\mathrm{VO}_{2} \max (\mathrm{l} / \mathrm{min}):$ Baseline } \\
\hline \multirow[t]{2}{*}{ Final } & -0.078 & 0.105 & 0.076 & 0.101 \\
\hline & 0.049 & $0.431^{*}$ & $0.413^{*}$ & $0.458^{*}$ \\
\hline \multicolumn{5}{|c|}{$\mathrm{VO}_{2} \max (\mathrm{ml} / \mathrm{kg} / \mathrm{min}):$} \\
\hline Baseline & 0.014 & 0.100 & 0.097 & 0.113 \\
\hline Final & 0.114 & $0.300^{*}$ & $0.248^{*}$ & $0.296^{*}$ \\
\hline \multicolumn{5}{|l|}{ BMI: } \\
\hline Baseline & 0.211 & 0.092 & 0.069 & 0.090 \\
\hline Final & 0.171 & -0.104 & -0.113 & -0.118 \\
\hline \multicolumn{5}{|c|}{$\%$ body fat: } \\
\hline Baseline: & 0.030 & -0.029 & -0.062 & -0.054 \\
\hline Final & 0.012 & -0.227 & -0.233 & $-0.250^{*}$ \\
\hline \multicolumn{5}{|l|}{$T G$} \\
\hline Baseline & 0.015 & $-0.251^{*}$ & -0.149 & -0.222 \\
\hline Final & $0.285^{*}$ & 0.015 & 0.015 & 0.016 \\
\hline \multicolumn{5}{|l|}{$H D L-C:$} \\
\hline Baseline & -0.228 & -0.007 & -0.154 & -0.101 \\
\hline Final & $-0.250^{*}$ & 0.003 & -0.029 & -0.015 \\
\hline \multicolumn{5}{|l|}{ SBP: } \\
\hline Baseline & 0.043 & 0.032 & 0.177 & 0.128 \\
\hline Final & 0.237 & 0.158 & 0.124 & 0.152 \\
\hline \multicolumn{5}{|l|}{$D B P:$} \\
\hline Baseline & -0.014 & -0.040 & 0.182 & 0.098 \\
\hline Final & 0.074 & 0.184 & 0.095 & 0.149 \\
\hline \multicolumn{5}{|l|}{ Glucose: } \\
\hline Baseline & 0.034 & -0.144 & 0.011 & -0.063 \\
\hline Final & -0.057 & -0.086 & -0.030 & -0.062 \\
\hline \multicolumn{5}{|l|}{ Insulin: } \\
\hline Baseline & 0.032 & -0.274 & $-0.382 *$ & $-0.432 *$ \\
\hline Final & 0.229 & -0.287 & -0.302 & -0.330 \\
\hline
\end{tabular}

$* p<0.05$.

plasma campesterol increased by $13 \%(\mathrm{p}=0.01)$, total plant sterols (sitosterol + campesterol) increased by $11 \%$ $(\mathrm{p}=0.03)$ and the ratio of campesterol to lathosterol increased by $13 \%(p=0.02)$ following the intervention. These data indicate that exercise training has no effect on cholesterol synthesis, but increases cholesterol absorption and the ratio between cholesterol absorption and synthesis.

Table 2 shows the correlation coefficients between plasma non-cholesterol sterols and metabolic syndrome risk factors at baseline and final testing. At baseline, campesterol was inversely related to plasma TG $(p=0.04)$, and sitosterol and total plant sterols were inversely correlated with fasting insulin levels $(p=0.04$ and $p=0.02$, respectively). At final testing, lathosterol was positively correlated with $\mathrm{TG}(\mathrm{p}=0.02)$, and inversely correlated with HDL-C $(\mathrm{p}=0.04)$. Furthermore, total plant sterols was inversely correlated with $\%$ body fat $(\mathrm{p}=0.04)$, while campesterol, sitosterol, and total plant sterols were positively correlated with $\mathrm{VO}_{2} \max$ whether expressed in absolute terms $(1 / \mathrm{min})(\mathrm{p}<0.05$ for each) or relative to body weight $(\mathrm{ml} / \mathrm{kg} / \mathrm{min})(\mathrm{p}<0.01$ for each).

The change in total plant sterol levels was inversely correlated with the change in body weight ( $\mathrm{r}=-0.271 ; \mathrm{p}=0.025$ ) (Fig. 1), and positively correlated with the change in $\mathrm{VO}_{2}$ max expressed in $1 / \mathrm{min}(\mathrm{r}=0.261$, $\mathrm{p}=0.036$ ). When $\mathrm{VO}_{2}$ was expressed relative to body 


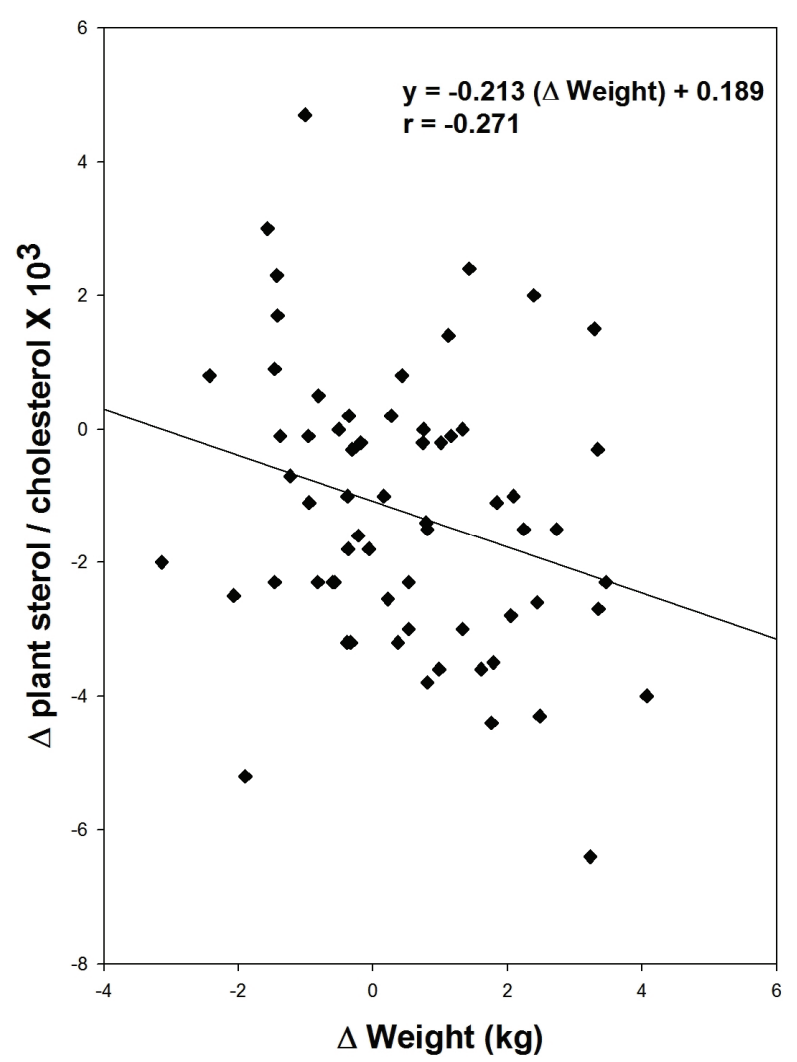

Fig. 1. Correlation between the change in plasma total plant sterols and body weight $(\mathrm{kg})$ after six months of endurance exercise training.

weight $(\mathrm{ml} / \mathrm{kg} / \mathrm{min})$ the correlation between the change in $\mathrm{VO}_{2} \mathrm{max}$ and plant sterols increased $(\mathrm{r}=0.350, \mathrm{p}=0.004)$ (Fig. 2), and was independent of changes in other metabolic syndrome risk factors in a stepwise multiple regression model (Table 3).

Diet records analyzed from a subset of subjects $(\mathrm{n}=30)$ showed that there was no difference in total cholesterol intake, total calories, or the percentage of calories from fat, protein, or carbohydrates at baseline and final testing $(\mathrm{p}<0.05$ for each). Furthermore, there was no correlation between changes in these dietary factors and changes in measures of body composition, plasma lipids or sterols ( $\mathrm{p}<0.05$ for each).

\section{Discussion}

The primary findings from this study include the following: 1) 6 months of endurance exercise training increased plasma levels of campesterol, total plant sterols (campesterol + sitosterol) and the ratio of campesterol to lathosterol; and 2) the change in total plant sterol level was positively correlated with the change in $\mathrm{VO}_{2} \max$, independent of the change in other metabolic syndrome

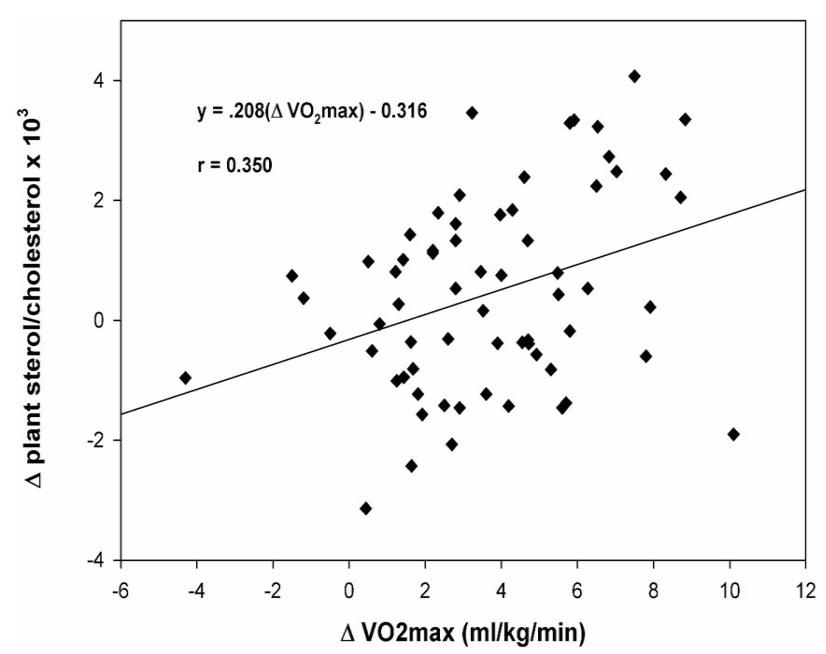

Fig. 2. Correlation between the change in plasma total plant sterols and $\mathrm{VO}_{2} \max (\mathrm{ml} / \mathrm{kg} / \mathrm{min})$ after six months of endurance exercise training.

Table 3. Results of multiple linear regression analysis for plant sterol levels as a function of $\mathrm{VO}_{2} \max (\mathrm{ml} / \mathrm{kg} / \mathrm{min})$ and metabolic syndrome risk factors.

\begin{tabular}{lccc}
\hline $\begin{array}{l}\text { Independent } \\
\text { variable }\end{array}$ & B & SE B & R \\
\hline $\begin{array}{l}\Delta \mathrm{VO}_{2} \\
\text { (ml/kg/min) }\end{array}$ & 0.205 & 0.076 & $0.346^{*}$ \\
$\begin{array}{l}\Delta \text { glucose } \\
\Delta S B P\end{array}$ & 0.028 & 0.026 & 0.188 \\
$\Delta D B P$ & 0.004 & 0.016 & 0.037 \\
$\triangle H D L-C$ & 0.013 & 0.023 & 0.075 \\
$\Delta T G$ & 0.001 & 0.035 & 0.005 \\
\hline & -0.002 & 0.006 & -0.039 \\
\hline
\end{tabular}

$* p<0.05$.

risk factors. Campesterol and sitosterol are markers of cholesterol absorption, while lathosterol is a marker of cholesterol synthesis (Miettinen et al. 1990). Thus these data suggests that exercise training increases cholesterol absorption and the ratio of cholesterol absorption to synthesis, whereas there was no significant change in cholesterol synthesis with training. Despite the increase in markers of cholesterol absorption, the plasma lipoprotein profile improved, as total and LDL-C levels were reduced, and HDL-C levels increased.

To our knowledge, this is the first study to demonstrate that long-term exercise training is associated with changes in these markers of cholesterol metabolism. Recently, Varady et al. (2004) showed that a shorter 8 -week endurance exercise program increased plasma 
lathosterol, but not plant sterol levels, and a follow-up study by the same group found that same 8 weeks of endurance exercise training had no effect on either cholesterol absorption or synthesis measured by the single stable isotope tracer method (Varady et al. 2007). There were several differences between our study and the studies by Varady et al. that may account for these discrepant findings. First, the length of the exercise intervention was significantly shorter in the studies by Varady et al. (8 weeks) compared to our study ( 24 weeks). It is possible that the adaptations that promote significant changes in cholesterol metabolism may not manifest themselves until after several months of exercise training. A second primary difference between these studies was the dietary control. In our study, all subjects were stabilized on an AHA step I diet prior to beginning the exercise intervention, while in the studies by Varady et al. subjects were only asked to maintain their current dietary regimens. It is possible that significant diet variations between subjects may have masked the effects of the exercise intervention on plant sterol levels or cholesterol absorption in their studies.

Another significant finding in our study was the correlations between plasma markers of cholesterol absorption with factors associated with the metabolic syndrome. At baseline, there was an inverse correlation between total plant sterol levels and fasting plasma insulin levels, and a trend for a correlation between these variables at final testing $(p=0.08)$. Furthermore, we found an inverse correlation between campesterol and TG levels at baseline, and an inverse correlation between $\%$ body fat and total plant sterols after the exercise training intervention. This is similar to the data of Simonen et al. (2000) who found a positive correlation between plant sterol levels and serum sex hormone binding globulin (SBHG), a marker of insulin sensitivity (Haffner 1996), and an inverse correlation between campesterol and TG levels after diet-induced weight loss. Several other studies have also noted inverse correlations between markers of cholesterol absorption and various metabolic syndrome risk factors (Gylling et al. 2004, Pihlajamaki et al. 2004), leading to speculation that low cholesterol absorption may be a component of the metabolic syndrome (Simonen et al. 2000). We believe our data provide additional support for this hypothesis.

Simonen et al. (2000) also found that the dietinduced change in plant sterols was inversely correlated to the change in body weight. In our study, the change in plant sterol levels was also inversely correlated with the change in body weight, but positively correlated with the change in $\mathrm{VO}_{2}$ max expressed in $1 / \mathrm{min}$. Furthermore, these variables contributed equally to the variation in plant sterol levels in a multiple regression model (data not shown). However, when $\mathrm{VO}_{2}$ was expressed relative to body weight $(\mathrm{ml} / \mathrm{kg} / \mathrm{min})$, the correlation between the change in plant sterols and $\mathrm{VO}_{2}$ max increased, and this correlation was independent of changes in other metabolic syndrome risk factors in a multiple regression analysis (Table 3). Many of the beneficial effects of exercise on risk factors for chronic disease are often attributed to weight loss induced by the increase in physical activity. Our data indicate that both weight loss and increases in cardiorespiratory fitness may have independent effects on cholesterol absorption.

The mechanisms responsible for the changes in markers of cholesterol absorption found in this study are uncertain. Simonen et al. (2000) hypothesized that increasing insulin resistance and obesity may change the intestinal cholesterol pool or the absorption mechanism of the intestinal mucosa. Thus the exercise could exert its effects on intestinal cholesterol absorption by improving insulin sensitivity. However, while insulin sensitivity was not measured in this study, we did not find a correlation between changes in plasma sterol levels and fasting insulin or glucose levels.

Another possibility is that changes in cholesterol metabolism could be due to alterations in the expression of genes involved in intestinal cholesterol transport. Cholesterol absorption is regulated by multiple genes expressed by enterocytes, including Niemann-Pick C1like1 (NPC1L1), which induces the influx of dietary cholesterol and plant sterols from the intestinal lumen into the enterocyte (Davis et al. 2004), and the ATP binding cassette transporters (ABC) G5 and G8, which limit sterol absorption by selectively pumping them back into the intestinal lumen (Yu et al. 2004). Several studies have shown that genetic polymorphisms in the ABCG5, ABCG8, and NPC1L1 genes affect cholesterol absorption in humans (Cohen et al. 2006, Gylling et al. 2004), and the variable expression of these genes may explain the differences in rates of cholesterol absorption between inbred strains of mice (Duan et al. 2004, 2006). Consequently, one way in which exercise may affect cholesterol absorption is by altering directly the intestinal expression of the ABCG5, ABCG8 or NPC-1L1 genes. In mice, we have shown that 3 months of endurance exercise training (treadmill running) reduced the expression of ABCG5, ABCG8, and NPC1L1 by $\sim 60 \%$ (Wilund et al. 2008). However, we are uncertain if exercise training 
modifies the expression of these genes in a similar manner in humans.

A positive correlation between cholesterol absorption and LDL-C levels has been seen in population studies (Kesaniemi and Miettinen 1987), suggesting that reducing cholesterol absorption may have therapeutic benefits. Furthermore, ezetemibe, bile acid sequestrants and dietary plant sterol intake are commonly used as therapies for reducing cholesterol absorption and lowering LDL-C levels. However, Simonen et al. (2000) showed that diet-induced weight loss increases cholesterol absorption, without increasing LDL-C levels, and our data presented here indicate that exercise training with modest weight loss also increases markers of cholesterol absorption, while decreasing LDL-C levels. This apparent paradox may be explained by data from earlier animal studies showing that endurance exercise training may increase the catabolism of cholesterol into bile acids (Hebbelinck and Casier 1966, Malinow et al. 1968) and the excretion of cholesterol and bile acids in the feces (Fukuda et al. 1979, Ostlund and Reaban 1989). As a result, increases in cholesterol absorption may be offset by increased catabolism and excretion of cholesterol, resulting in no change or the modest reductions in LDL-C seen in many exercise training studies.

There are several limitations to this study. The first is the absence of a non-exercising control group. The subjects analyzed here were part of a larger trial in which subjects with polymorphic variations at specific gene loci served as the comparison groups, so a sedentary control group was not included. Second, we did not measure plasma variables prior to beginning the 6-week dietary stabilization period, so we cannot exclude the possibility that the changes in plasma sterols were influenced by the change in diet prior to beginning the exercise intervention. However, we believe that the 6-week lead-in-period for the dietary changes makes this unlikely. Future studies using randomized controlled trial designs should be conducted to confirm the results presented here. A third limitation of this study was that we estimated cholesterol absorption and synthesis using plasma markers (plant sterols and lathosterol, respectively), as opposed to using stable isotope methods to directly measure cholesterol metabolism. Though not as precise as measuring isotope kinetics, estimating cholesterol absorption and synthesis by measuring plasma sterol measurements represent a less expensive and much simpler method of estimating these variables that has been used extensively in recent epidemiological studies.

In conclusion, we found that endurance exercise training increased markers of cholesterol absorption, but did not affect markers of cholesterol synthesis, in elderly individuals with at least one metabolic syndrome risk factor. Despite this, the lipoprotein-lipid profile was improved, as there was a reduction in TG, TC, and LDL$\mathrm{C}$, and an increase in HDL-C following the intervention. This exercise-induced increase in cholesterol absorption may indicate a correction in cholesterol metabolism in this population and highlights the complex relationship between chronic physical activity and cholesterol homeostasis.

\section{Conflict of Interest}

There is no conflict of interest.

\section{Acknowledgements}

This research was supported by grants No. AG-00268, AG-15389, and AG-17474 from the National Institutes of Health, and a pilot grant from the University of Illinois Campus Research Board.

\section{References}

CHAN DC, WATTS GF, BARRETT PH, O'NEILL FH, THOMPSON GR: Plasma markers of cholesterol homeostasis and apolipoprotein B-100 kinetics in the metabolic syndrome. Obes Res 11: 591-596, 2003.

COHEN JC, PERTSEMLIDIS A, FAHMI S, ESMAIL S, VEGA GL, GRUNDY SM, HOBBS HH: Multiple rare variants in NPC1L1 associated with reduced sterol absorption and plasma low-density lipoprotein levels. Proc Natl Acad Sci U S A 103: 1810-1815, 2006.

DAVIS HR JR, ZHU LJ, HOOS LM, TETZLOFF G, MAGUIRE M, LIU J, YAO X, IYER SP, LAM MH, LUND EG, DETMERS PA, GRAZIANO MP, ALTMANN SW: Niemann-Pick C1 Like 1 (NPC1L1) is the intestinal phytosterol and cholesterol transporter and a key modulator of whole-body cholesterol homeostasis. J Biol Chem 279: 33586-33592, 2004.

DUAN LP, WANG HH, OHASHI A, WANG DQ: Role of intestinal sterol transporters Abcg5, Abcg8, and Npc111 in cholesterol absorption in mice: gender and age effects. Am J Physiol 290: G269-G276, 2006. 
DUAN LP, WANG HH, WANG DQ: Cholesterol absorption is mainly regulated by the jejunal and ileal ATP-binding cassette sterol efflux transporters Abcg5 and Abcg8 in mice. J Lipid Res 45: 1312-1323, 2004.

FUKUDA N, IDE T, KIDA Y, TAKAMINE K, SUGANO M: Effects of exercise on plasma and liver lipids of rats. IV. Effects of exercise on hepatic cholesterogenesis and fecal steroid excretion in rats. Nutr Metab 23: 256-265, 1979.

GYLLING H, HALLIKAINEN M, PIHLAJAMAKI J, AGREN J, LAAKSO M, RAJARATNAM RA, RAURAMAA R, MIETTINEN TA: Polymorphisms in the ABCG5 and ABCG8 genes associate with cholesterol absorption and insulin sensitivity. J Lipid Res 45: 1660-1665, 2004.

GYLLING H, MIETTINEN TA: Cholesterol absorption, synthesis, and LDL metabolism in NIDDM. Diabetes Care 20: 90-95, 1997.

HAFFNER SM: Sex hormone-binding protein, hyperinsulinemia, insulin resistance and noninsulin-dependent diabetes. Horm Res 45: 233-237, 1996.

HEBBELINCK M, CASIER H: Effect of muscular exercise on the metabolism of 4-C14 labelled cholesterol in mice. Int $Z$ Angew Physiol 22: 185-189, 1966.

KESANIEMI YA, MIETTINEN TA: Cholesterol absorption efficiency regulates plasma cholesterol level in the Finnish population. Eur J Clin Invest 17: 391-395, 1987.

MALINOW MR, MCLAUGHLIN P, PERLEY A: Cholesterol: treadmill activity accelerates oxidation in rats. Science 160: 1239-1240, 1968.

MAZESS RB, BARDEN HS, BISEK JP, HANSON J: Dual-energy x-ray absorptiometry for total-body and regional bone-mineral and soft-tissue composition. Am J Clin Nutr 51: 1106-1112, 1990.

MIETTINEN TA, GYLLING H, TUOMINEN J, SIMONEN P, KOIVISTO V: Low synthesis and high absorption of cholesterol characterize type 1 diabetes. Diabetes Care 27: 53-58, 2004.

MIETTINEN TA, TILVIS RS, KESANIEMI YA: Serum plant sterols and cholesterol precursors reflect cholesterol absorption and synthesis in volunteers of a randomly selected male population. Am J Epidemiol 131: 20-31, 1990.

NICKLAS BJ, ROGUS EM, COLMAN EG, GOLDBERG AP: Visceral adiposity, increased adipocyte lipolysis, and metabolic dysfunction in obese postmenopausal women. Am J Physiol 270: E72-E78, 1996.

OSTLUND RE JR, REABAN M: Effect of exercise training on plasma cholesterol and cholesterol kinetics in adult female rats. Atherosclerosis 75: 7-11, 1989.

PIHLAJAMAKI J, GYLLING H, MIETTINEN TA, LAAKSO M: Insulin resistance is associated with increased cholesterol synthesis and decreased cholesterol absorption in normoglycemic men. J Lipid Res 45: 507-512, 2004.

SEALS DR, HAGBERG JM, HURLEY BF, EHSANI AA, HOLLOSZY JO: Endurance training in older men and women. I. Cardiovascular responses to exercise. J Appl Physiol 57: 1024-1029, 1984.

SIMONEN P, GYLLING H, HOWARD AN, MIETTINEN TA: Introducing a new component of the metabolic syndrome: low cholesterol absorption. Am J Clin Nutr 72: 82-88, 2000.

SUTHERLAND WH, SCOTT RS, LINTOTT CJ, ROBERTSON MC, STAPELY SA, COX C: Plasma non-cholesterol sterols in patients with non-insulin dependent diabetes mellitus. Horm Metab Res 24: 172-175, 1992.

VARADY KA, EBINE N, VANSTONE CA, PARSONS WE, JONES PJ: Plant sterols and endurance training combine to favorably alter plasma lipid profiles in previously sedentary hypercholesterolemic adults after 8 wk. Am J Clin Nutr 80: 1159-1166, 2004.

VARADY KA, HOUWELING AH, JONES PJ: Effect of plant sterols and exercise training on cholesterol absorption and synthesis in previously sedentary hypercholesterolemic subjects. Transl Res 149: 22-30, 2007.

WILUND KR, COLVIN PL, PHARES D, GOLDBERG AP, HAGBERG JM: The effect of endurance exercise training on plasma lipoprotein AI and lipoprotein AI:AII concentrations in sedentary adults. Metabolism 51: 1053-1060, 2002.

WILUND KR, FEENEY LA, TOMAYKO EJ, CHUNG HR, KIM K: Endurance exercise training reduces gallstone development in mice. J Appl Physiol 104: 761-765, 2008.

WILUND KR, YU L, XU F, VEGA GL, GRUNDY SM, COHEN JC, HOBBS HH: No association between plasma levels of plant sterols and atherosclerosis in mice and men. Arterioscler Thromb Vasc Biol 24: 2326-2332, 2004.

YU L, VON BERGMANN K, LUTJOHANN D, HOBBS HH, COHEN JC: Selective sterol accumulation in ABCG5/ABCG8-deficient mice. J Lipid Res 45: 301-307, 2004. 